Article

\title{
Green Route for the Isolation and Purification of Hyrdoxytyrosol, Tyrosol, Oleacein and Oleocanthal from Extra Virgin Olive Oil
}

\author{
Antonio Francioso ${ }^{1, *(\mathbb{D})}$, Rodolfo Federico ${ }^{2}$, Anna Maggiore ${ }^{1}$, Mario Fontana ${ }^{1}{ }^{1}$, \\ Alberto Boffi ${ }^{1,2}$, Maria $D^{\prime}$ Erme $^{1}$ and Luciana Mosca ${ }^{1}$ (D) \\ 1 Department of Biochemical Sciences, "Sapienza” University of Rome, Piazzale Aldo Moro 5, 00185 Rome, \\ Italy; anna.maggiore@uniroma1.it (A.M.); mario.fontana@uniroma1.it (M.F.); \\ alberto.boffi@uniroma1.it (A.B.); maria.derme@uniroma1.it (M.D.); luciana.mosca@uniroma1.it (L.M.) \\ 2 MOLIROM s.r.l, via Carlo Bartolomeo Piazza 8, 00161 Rome, Italy; r.federico@molirom.com \\ * Correspondence: antonio.francioso@uniroma1.it; Tel.: +39-06-4991-0987
}

Academic Editors: Rafael Guillén Bejarano and María Rocío Rodríguez Arcos Received: 6 July 2020; Accepted: 10 August 2020; Published: 11 August 2020

\begin{abstract}
Extra virgin olive oil (EVOO) phenols represent a significant part of the intake of antioxidants and bioactive compounds in the Mediterranean diet. In particular, hydroxytyrosol (HTyr), tyrosol (Tyr), and the secoiridoids oleacein and oleocanthal play central roles as anti-inflammatory, neuro-protective and anti-cancer agents. These compounds cannot be easily obtained via chemical synthesis, and their isolation and purification from EVOO is cumbersome. Indeed, both processes involve the use of large volumes of organic solvents, hazardous reagents and several chromatographic steps. In this work we propose a novel optimized procedure for the green extraction, isolation and purification of HTyr, Tyr, oleacein and oleocanthal directly from EVOO, by using a Natural Deep Eutectic Solvent (NaDES) as an extracting phase, coupled with preparative high-performance liquid chromatography. This purification method allows the total recovery of the four components as single pure compounds directly from EVOO, in a rapid, economic and ecologically sustainable way, which utilizes biocompatible reagents and strongly limits the use or generation of hazardous substances.
\end{abstract}

Keywords: extra virgin olive oil; oleocanthal; secoiridoids; green chemistry; hydoxytyrosol; tyrosol; oleacein; natural deep eutectic solvents; ultra-high-performance liquid chromatography; mass spectrometry

\section{Introduction}

Extra virgin olive oil (EVOO) represents one of the most important sources of biologically active compounds and antioxidants in the Mediterranean diet [1-5]. EVOO polyphenols and secoiridoids possess important biological effects, and are extremely interesting and promising compounds from a pharmacological point of view [3,6-8]. Hydroxytyrosol (HTyr), tyrosol (Tyr), oleacein and oleocanthal are the main compounds responsible for the beneficial effects of EVOO as part of the Mediterranean $\operatorname{diet}$ (Figure 1) $[9,10]$.

These compounds possess a strong antioxidant activity, and HTyr and oleocanthal in particular have a synergistic marked anti-inflammatory effect [11-14]. Tyr instead possesses important central nervous system and cardioprotective effects [9,15-20]. Oleocanthal and its less studied cathecolic analog, oleacein, are nowadays two of the most interesting bioactive natural products under biological investigation [10]. Oleocanthal is responsible for the particular spicy organoleptic sensation that EVOO induces on the tongue. The scientific relevance of and interest in oleocanthal is mainly due to its potent anti-inflammatory effect (several times more active than other FANS, e.g., Ibuprofen), its selective 
cytotoxicity for cancer cells, and the very promising neuroprotective activity that oleocanthal has demonstrated in neurodegenerative pathologies, i.e., Alzheimer's disease [6,7,13,14,21-27]. Given all these considerations, these compounds are extremely interesting and promising for the preparation of dietary supplements, nutraceuticals, functional foods or cosmeceuticals.<smiles>OCCc1ccc(O)c(O)c1</smiles><smiles>C/C=C(/C=O)C(CC=O)CC(=O)OCCc1ccc(O)c(O)c1</smiles>

Oleacein<smiles>OCCc1ccc(O)cc1</smiles><smiles>C/C=C(/C=O)C(CC=O)CC(=O)OCCc1ccc(O)cc1</smiles>

Oleocanthal

Despite their promising pharmacological activities, there is still a limited number of studies that have been carried out on oleocanthal and oleacein, as these molecules are not easily available on the market, and even when available are extremely expensive. The difficulty of obtaining large amounts of pure compounds at affordable prices is principally due to their expensive and difficult purification from EVOO. Oleocanthal and oleacein are not present in Olea europaea L. plants (leaves and fruits), but are formed during EVOO production and processing via the conversions of oleuropein and ligstroside, two glycosidic secoiridoids derived from HTyr and Tyr, respectively (Figure 2) [16,28-32].

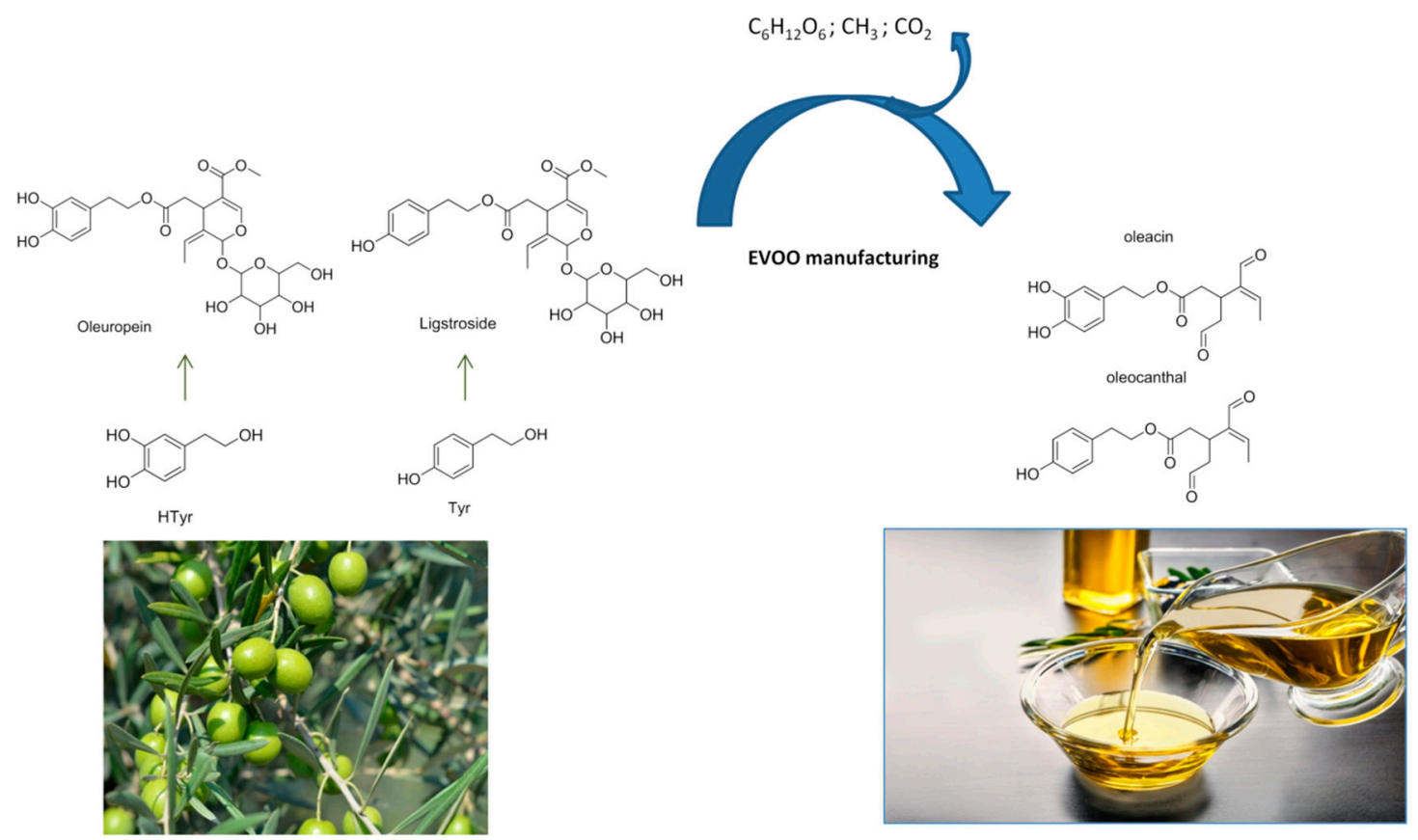

Figure 2. Oleocanthal and oleacein formation. During EVOO manufacturing, secoiridoids (oleuropein and ligstroside) are deglycosylated, and after demethylation and spontaneous decarboxylation free dialdehydes are produced.

The isolation of oleocanthal and oleacein requires the extensive treatment of EVOO with organic solvents such as $n$-hexane and acetonitrile, and subsequently several chromatographic separation steps (mostly silica based) and the use of organic eluents such as dichloromethane, petroleum ether and ethyl acetate [33-36]. Even by using large volumes of organic solvents in the extraction, the gravity 
columns and the preparative thin layer chromatography, the yields and the purity of the products are often low, expensive, and moreover not ecologically friendly and biocompatible, and do not eliminate the use or generation of hazardous substances. With this work, we propose a novel strategy for the green extraction and isolation of HTyr, Tyr, oleacein and oleocanthal in a single chromatographic step, using natural deep eutectic solvent (NaDES) extraction coupled with preparative high-performance liquid chromatography. Our method allows an efficient and complete extraction of the aforementioned compounds, obtaining highly concentrated, high final yields of the pure products with the use of biocompatible reagents, leaving no hazardous organic residues after the purification.

\section{Results}

\subsection{Phenols and Secoiridoids Characterization in EVOO/NaDES Extract}

Our results show that EVOO treatment with NaDES (Choline:Glycerol, 1:1.5 molar ratio) allowed us to obtain highly concentrated solutions of HTyr, Tyr, oleacein and oleocanthal by extracting them directly with a 1:20 ratio $(w / w)$ of NaDES:EVOO. The UPLC-PDA/MS analysis of EVOO NaDES extracts revealed the presence of HTyr, Tyr, oleacein and oleocanthal as major components, together with other minor products eluted in the last part of the hydrophobic chromatographic gradient (Figure 3). Compounds were identified by their retention time, UV-visible and MS spectra, and by comparison with pure analytical standards.

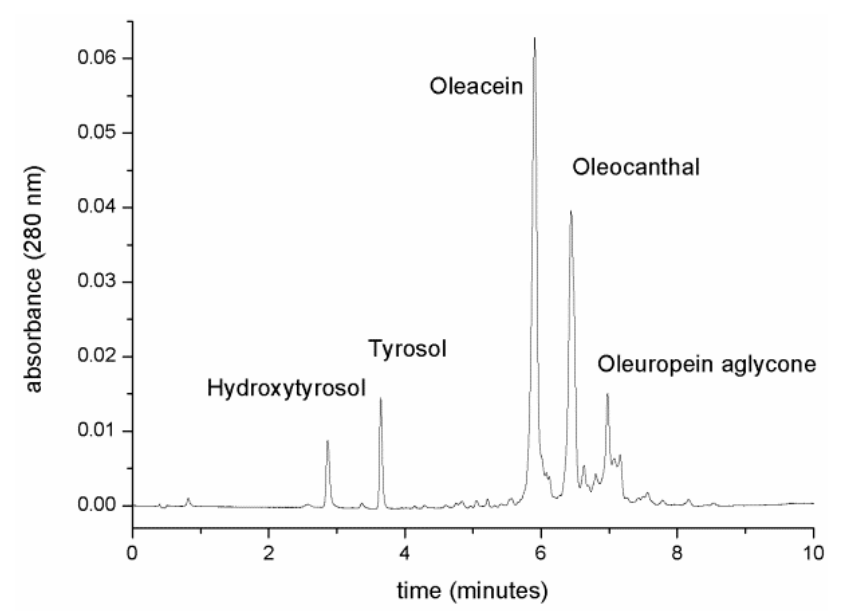

Figure 3. UPLC chromatographic analysis of EVOO/NaDES extract.

As shown by the UPLC chromatogram (Figure 3), the first two eluted compounds were identified as HTyr and Tyr, with retention times of 2.8 and $3.6 \mathrm{~min}$, respectively. The identity of these compounds was verified by the analysis of their UV-visible spectra, and by the injection of the analytical standards. The MS spectra under our conditions were not available due to the lower ionization features of these two phenols, with respect to their secoiridoids derivatives. The catechol moiety of HTyr has a characteristic UV-visible spectrum, with a $\lambda_{\max }$ at $280 \mathrm{~nm}$, whereas the mono substituted phenol Tyr displays a UV-visible $\lambda_{\max }$ at a lower wavelength $(275 \mathrm{~nm})$, with a shoulder at $280 \mathrm{~nm}$ (Figure 4).

The oleacein and oleocanthal in NaDES extracts were recognized firstly by analysis of their UV-visible and MS spectra, which was then confirmed by analytical standard injection. These two phenolic secoiridoids possess the same chromophoric moiety as their precursors (catechol for HTyr and phenol for Tyr), with the same UV-visible spectra and different characteristic MS negative ionization spectra (Figure 5). Oleacein elutes at $5.9 \mathrm{~min}$, and its pseudo-molecular ion displays an $[\mathrm{M}-\mathrm{H}]^{-}$peak at $319.08 \mathrm{~m} / \mathrm{z}$. Oleocanthal (retention time, $6.4 \mathrm{~min}$ ) possess the same UV-visible spectrum as Tyr, with a pseudomolecular ion $[\mathrm{M}-\mathrm{H}]^{-}$at $303.08 \mathrm{~m} / \mathrm{z}$ in the same negative ESI-MS mode. 

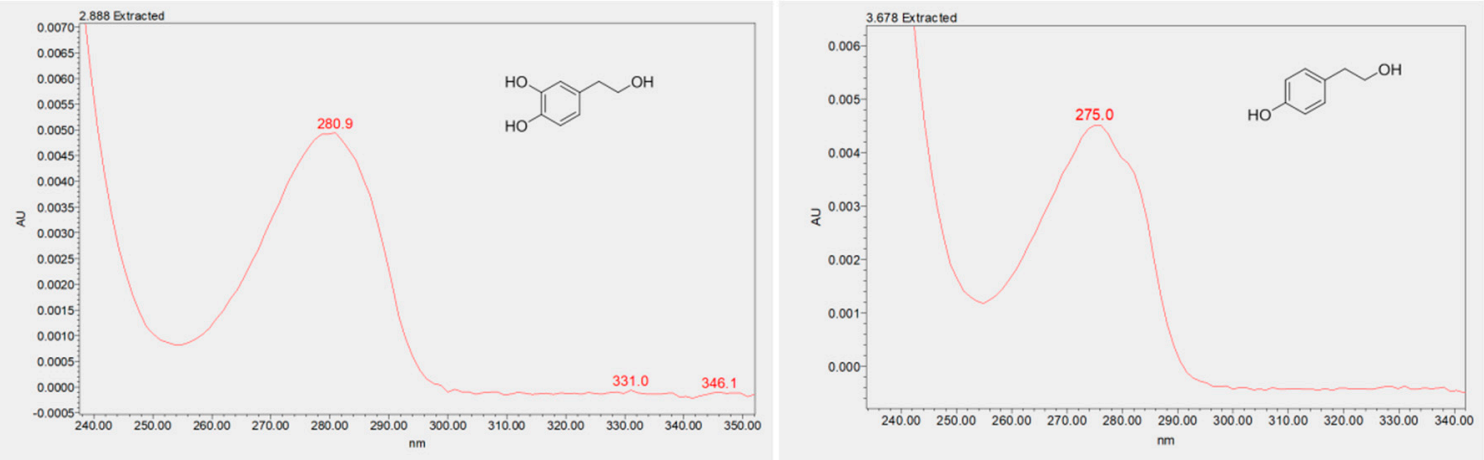

Figure 4. HTyr and Tyr UV-visible spectra.
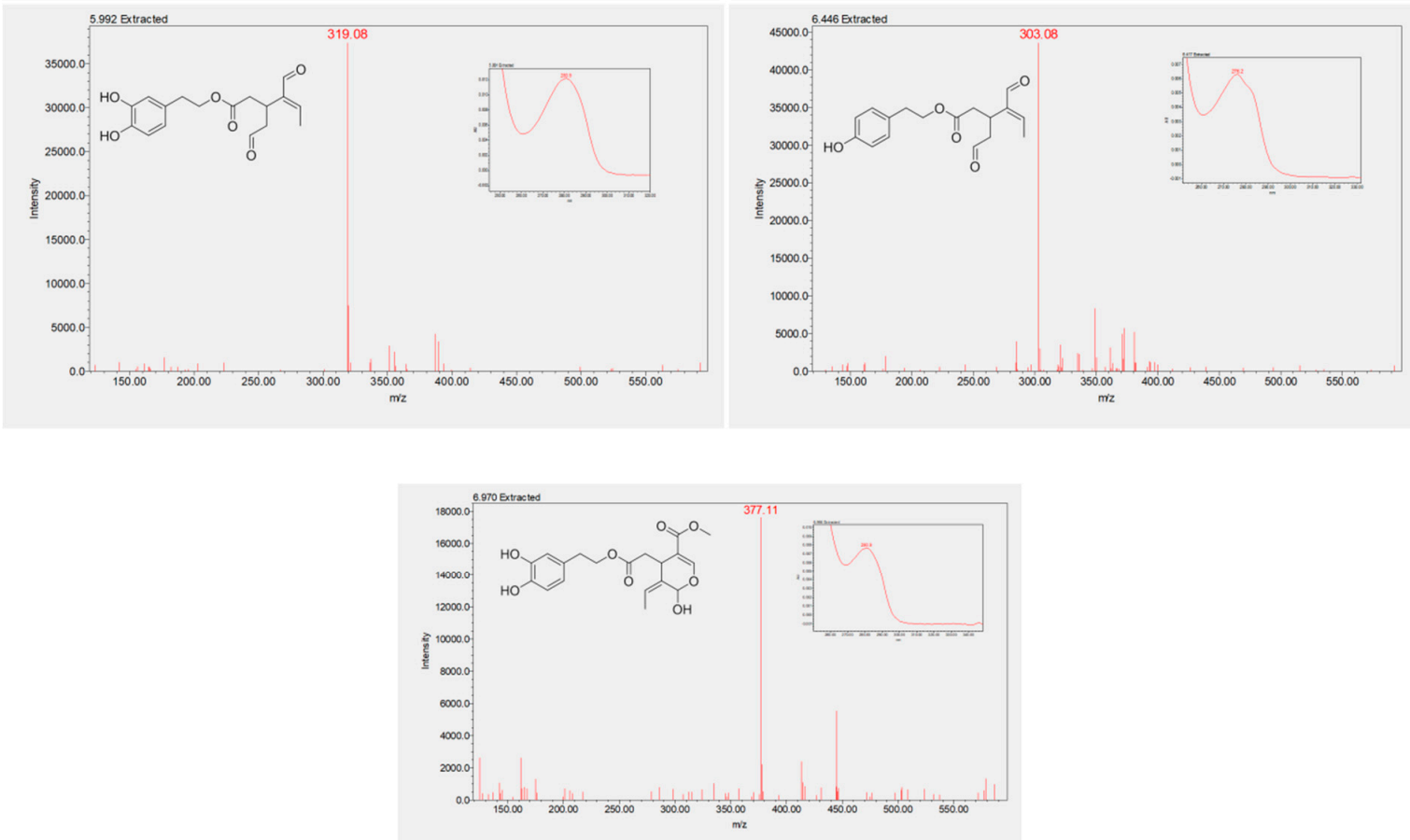

Figure 5. Oleacein (left), oleocanthal (right) and oleuropein aglycone (down) $\mathrm{MS}^{-}$and UV-visible spectra (inset).

Though not of interest in this work, another minor compound eluted at $6.9 \mathrm{~min}$ (Figure 2) was detected, and tentatively characterized by means of chromatographic retention factor, UV-visible spectrum and MS spectrum. The compound displays the UV-visible features of EVOO catechols, and an $[\mathrm{M}-\mathrm{H}]^{-}$pseudomolecular ion at $377.11 \mathrm{~m} / \mathrm{z}$, as reported in the literature for oleuropein aglycone [37-39](Figure 5).

\subsection{Extraction, Determination and Compounds Isolation}

EVOO was extracted three times with NaDES, and after each extraction step the compounds of interest were determined and quantified in the extracts.

The UPLC chromatographic profiles and calibration curves of the HTyr, Tyr, oleacein and oleocanthal analytical standards are shown in Figure 6. 

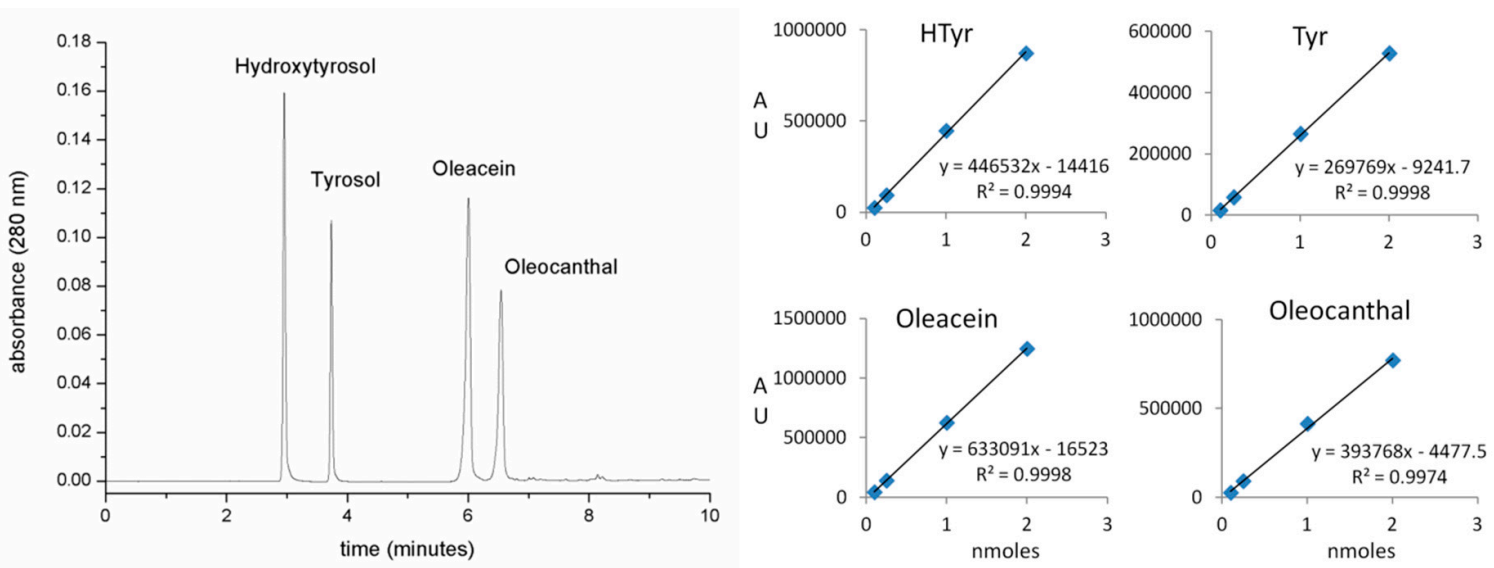

Figure 6. UPLC representative chromatogram (left) and calibration curves (right) of phenols and secoiridoids analytical standards.

After the first extraction, EVOO was re-extracted two more times with the same ratio of NaDES:EVOO $(1: 20 w / w)$ to evaluate the efficiency of the extraction and the relative recovery of the single compounds after each step. Our results show that the first extraction allows the recovery of almost all of the four compounds, with almost 100\% yield for the catechol compounds (HTyr and oleacein; $100 \%$ and $97 \%$, respectively) and a slightly lower yield for Tyr and oleocanthal $(90 \%$ and $70 \%$, respectively). The second extraction recovers the remaining amount of all the compounds, and the last one allows the complete recovery of the last residues of oleocanthal (Figure 7).

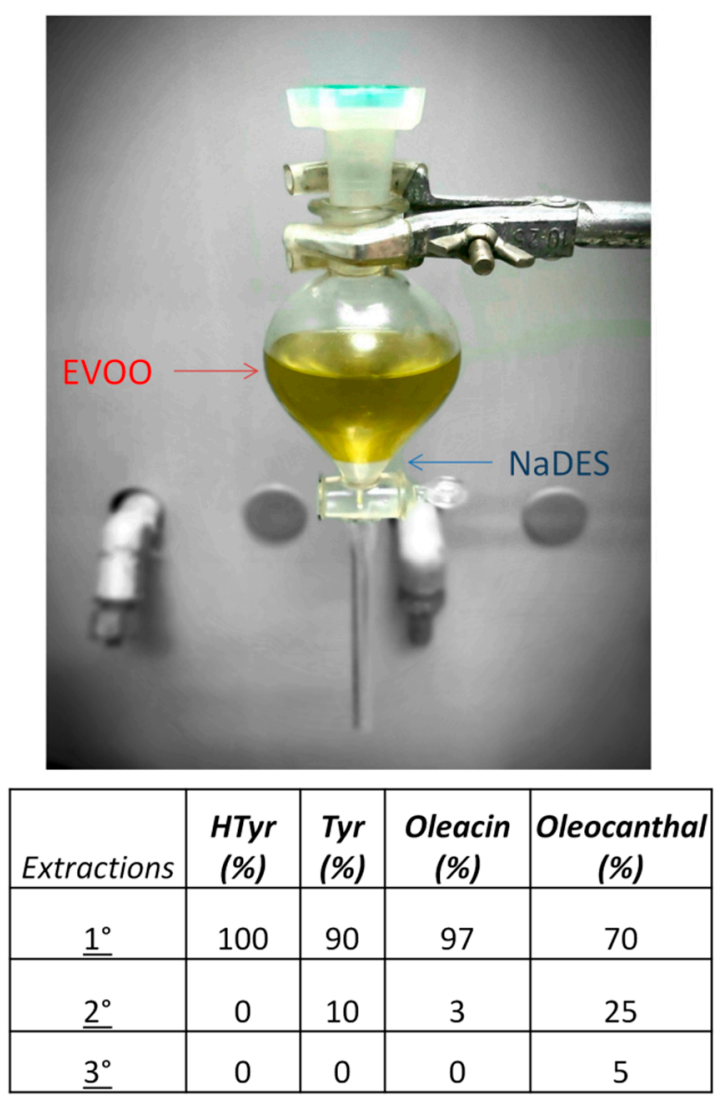

Figure 7. EVOO extraction with NaDES (up) and percentage recovery of the compounds after each extraction step (bottom). 
The first extract was purified via reverse phase preparative HPLC. The second and the third extracts were combined together and purified by the same process. Before injection, each extract was added to a 1.5 volume of ultrapure water (1:1.5 extract:water) to permit feasible injection with no significant increase of backpressure caused by the high viscosity of NaDES compared to pure water. The elution was performed with an ethanol gradient in ultrapure water and, as shown in Figure 8 , the peak selectivity and chromatographic behavior were maintained at the same levels as those of the UPLC analytical reverse phase system.

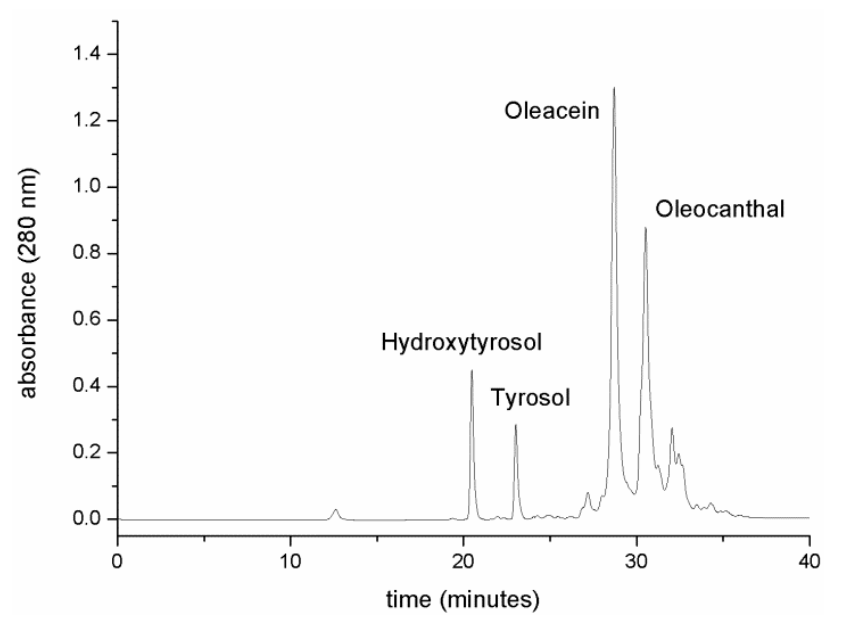

Figure 8. Reverse phase preparative HPLC purification of the compounds from NaDES extracts.

The peaks of interest were collected and the solvent was reduced by rotary evaporation. Dry pure compounds were then recovered from aqueous elutes by freeze-drying. After the overall purification process, from $1 \mathrm{~kg}$ of EVOO we obtained ca. $102 \mathrm{mg}$ of oleocanthal, $82 \mathrm{mg}$ of oleacein, $11 \mathrm{mg}$ of Tyr and $9 \mathrm{mg}$ of HTyr.

\section{Discussion}

With this work we developed and set up a novel method for the purification of HTyr, Tyr, oleacein and oleocanthal from EVOO, using a completely green and simple two step procedure. Previous papers reported the extraction of EVOO polyphenols using deep eutectic solvents for the analytical determination of EVOO polyphenols, but no studies were carried out on the application and optimization of the extraction and preparative purification of these four compounds [40-44]. NaDES extraction allowed us to obtain highly concentrated green solutions of HTyr, Tyr, oleacein and oleocanthal, which were identified and determined by analytical standards authentication and by means of UPLC-DAD/MS. The chromatographic analysis evidenced that catechol compounds (HTyr and oleacein) both elute before their respective mono substituted phenolic analogues (Tyr and oleocanthal), due to their more hydrophilic nature and the minor retention factors in reverse stationary phases. The actual method involves three subsequent extractions of EVOO, which afford a total recovery of the molecules, with different relative amounts of each compound at each step. The first extraction leads to the recovery of almost all the simple phenols (HTyr and Tyr) and oleacein, and about $70 \%$ of the oleocanthal. The remaining part of oleocanthal is purified by pooling together the second and the third extractions, in which the compound is derived as the major constituent. The strategy of using very concentrated NaDES phenolics and secoirodoids extracts gives several advantages. The first one is the obtainment of highly concentrated preparations in a non-volatile medium that can be further easily processed. The second advantage is the different relative extraction power of NaDES in the given ratio with EVOO (1:20 w/w), which allows an enrichment of the first extract that improves the subsequent chromatographic separation steps. The third and the most interesting feature of this methodology is the possibility of using reverse phase preparative HPLC for the purification of such 
concentrated compounds in solution. Phenolics and secoiridoids are not such hydrophilic compounds, and the injection phase of these molecules into preparative RP-HPLC is often a problem. The use of NaDES aqueous extracts (1:1.5 extract:water)in the injection phase maintains the compounds in the hydrophilic phase, with no significant influence on solubility and injection, and no backpressure problems caused by the high viscosity of pure NaDES with respect to the initial mobile phase (water). With this method it is possible to purify ca. $200 \mathrm{mg}$ of oleocanthal and oleacein from $1 \mathrm{~kg}$ of EVOO, with few RP-preparative HPLC chromatographic purifications and with a total recovery of the four compounds in a completely green process; a yield that, so far, is at least two times higher than that achieved by other reported methods [33,45]. As regards the phenolics precursors HTyr and Tyr, their amounts and concentrations in different EVOO depends not only on the Olea Europea L. cultivar, but also on the aging of the EVOO. It is even possible with this method to obtain higher yields of HTyr and Tyr by applying the purification process to aged EVOOs, in which the secoiridoids are converted back into their precursors over time. Our method finally gives the opportunity to many research laboratories to purify such scientifically interesting and very expensive compounds, via a completely green and efficient method, allowing the possibility of further studies in the biological, chemical and biochemical field.

\section{Materials and Methods}

\subsection{Reagents and Chemicals}

All the reagents and chemicals were purchased, at analytical grade, from Sigma-Aldrich (Milan, Italy). HPLC and LC-MS grade solvents used were purchased from Carlo Erba (Milan, Italy). HTyr, Tyr, oleacein and oleocanthal analytical standards were purchased from Sigma-Merck (Darmstadt, Germany). EVOO (Olea Europea L., Coratina cultivar) was obtained from Puglia region (Italy).

\subsection{UPLC-DAD/MS}

UPLC-DAD/MS was performed on a Waters Acquity H-Class UPLC system (Waters, Milford, MA, USA), including a quaternary solvent manager (QSM), a sample manager with a flow through needle system (FTN), a photodiode array detector (PDA) and a single-quadruple mass detector with electrospray ionization source (ACQUITY QDa). Chromatography was performed on a Phenomenex Kinetex C18 column (100 $\mathrm{mm} \times 2.1 \mathrm{~mm}$ i.d., $2.6 \mu \mathrm{m}$ particle size). Solvent A was $0.1 \%$ aqueous $\mathrm{HCOOH}$ and solvent $\mathrm{B}$ was $0.1 \% \mathrm{HCOOH}$ in $\mathrm{MeOH}$. The flow rate was $0.5 \mathrm{~mL} / \mathrm{min}$ and column temperature was set at $35{ }^{\circ} \mathrm{C}$. Elution was performed isocratically for the first minute with $2 \% \mathrm{~B}$; from minute 1 to minute 6 solvent B was linearly increased to 55\%; from 6 to $10 \mathrm{~min} 20 \% \mathrm{~A}$ and 80\% B; then, for $0.5 \mathrm{~min}$ solvent B was set at $100 \%$ and maintained for $2 \mathrm{~min}$. The column was re-equilibrated with $98 \% \mathrm{~A}$ and $2 \%$ B before the next injection. Samples were diluted in the mobile phase and injected through the needle. The PDA detector was set up in the range 200 to $600 \mathrm{~nm}$. Mass spectrometric detection was performed in the negative electrospray ionization mode, using nitrogen as the nebulizer gas. Analyses were performed in the Total Ion Current (TIC) mode with a mass range of $50-1000 \mathrm{~m} / \mathrm{z}$. The capillary voltage was $0.8 \mathrm{kV}$, cone voltage $15 \mathrm{~V}$, ion source temperature $120^{\circ} \mathrm{C}$ and probe temperature $600{ }^{\circ} \mathrm{C}$. Quantification of each compound was performed by using standards calibration curves in the range of 0.1-2 nmol in the column.

\subsection{EVOO Extraction and Compounds Isolation}

NaDES choline:glycerol (1:1.5 molar ratio) was prepared by mixing the two components at $60{ }^{\circ} \mathrm{C}$ under magnetic stirring for $1 \mathrm{~h}$. After cooling EVOO was added to the NaDES in the ratio 1:20 w/w (NaDES:EVOO). The extractions were carried out under magnetic stirring at room temperature for $15 \mathrm{~min}$ and then transferred via a separatory funnel for decantation and phases separation. The same procedure was repeated 3 times to evaluate the recovery of single compounds after each extraction. After UPLC quantification of the phenolic and secoridoid components, the second and 
the third extractions were pooled together and these new extracts were each added in a ratio of 1.5 to bidistilled water. These two NaDES aqueous extracts were subsequently purified by preparative HPLC. Preparative chromatography was performed on a Waters HPLC 600 pump equipped with a controller and an UV-Vis photodiode array detector mod. 2996. The chromatographic column was a reverse phase Vydac C18 column, $22 \mathrm{~mm} \times 250 \mathrm{~mm}, 10 \mu \mathrm{m}$ particle size. Data analysis was monitored using a dedicated application $\left(\right.$ Millennium ${ }^{32}$ ). Elution was performed at $8 \mathrm{~mL} / \mathrm{min}$ with a binary gradient system with bidistilled water (solvent A) and 80\% aqueous ethanol (solvent B). The gradient was: $0-5 \mathrm{~min}, 2 \% \mathrm{~B} ; 5-35 \mathrm{~min}, 100 \% \mathrm{~B}$. During the separation the products were monitored spectrophotometrically at $280 \mathrm{~nm}$ and directly collected from the detector. After compound collection the solvents were evaporated under reduced pressure using a rotary evaporator, and finally dried by freeze-drying.

\section{Conclusions}

This work demonstrates the possibility of using a convenient, rapid and totally green novel methodology for the purification of HTyr, Tyr, oleacein and oleocanthal from EVOO by direct NaDES extraction, obtaining highly concentrated solutions of phenols and secoiridoids. These solutions possess several advantages with respect to classical organic extracts, especially for reverse phase preparative HPLC applications. The hydrophilic nature of NaDES allows its injection at large volumes into the starting reverse phase gradients, with no influence on the resolution and selectivity of the chromatography. As mentioned above, the present method, with respect to the currently available procedures, represents a completely green alternative for the isolation of EVOO phenolics and secoiridoids, which provides the total recovery of the compounds and prevents degradation processes caused by extensive organic extractions and different separation steps. Moreover, besides being an eco-friendly process, the proposed purification is even economically sustainable compared to traditional methods, in which large amounts of organic solvents are utilized and low yields and purities of the compounds are obtained. Due to the high costs and the pharmacological potential that EVOO phenols, and especially secoiridoids such as oleocanthal, display, the present method will represent a new solution for scientists who want to further investigate the chemical and biological properties of these natural products, of which there is still much to explore.

Author Contributions: Data curation, M.D., A.B., A.M.; Writing-original draft preparation, A.F., R.F. and L.M.; Writing-review and editing, A.F., R.F., L.M., A.B. and M.D.; Visualization, A.F. and A.M.; Supervision, M.F., R.F. and L.M.; Project administration, A.F., R.F. and L.M.; Funding acquisition, L.M., A.B. and R.F. All authors have read and agreed to the published version of the manuscript.

Funding: This research was funded by Ateneo 2018 and Ateneo 2019 grants funded by “Sapienza" University of Rome to A.F. and L.M.

Acknowledgments: Authors want to acknowledge Alessandra Franco for her technical support and assistance.

Conflicts of Interest: The authors declare no conflict of interest

\section{References}

1. Tresserra-Rimbau, A.; Lamuela-Raventós, R.M. Olives and Olive Oil: A mediterranean source of polyphenols. In Olives and Olive Oil as Functional Foods; John Wiley \& Sons, Ltd.: Chichester, UK, 2017; pp. 417-434.

2. Servili, M.; Sordini, B.; Esposto, S.; Urbani, S.; Veneziani, G.; Di Maio, I.; Selvaggini, R.; Taticchi, A. Biological activities of phenolic compounds of extra virgin olive oil. Antioxidants 2013, 3, 1-23. [CrossRef] [PubMed]

3. Ramírez-Anaya,J.D.P.; Samaniego-Sánchez,C.; Castañeda-Saucedo, M.C.; Villalón-Mir, M.; de la Serrana, H.L.-G. Phenols and the antioxidant capacity of mediterranean vegetables prepared with extra virgin olive oil using different domestic cooking techniques. Food Chem. 2015, 188, 430-438. [CrossRef] [PubMed]

4. Ramírez-Anaya, J.d.P.; Castañeda-Saucedo, M.C.; Olalla-Herrera, M.; Villalón-Mir, M.; de la Serrana, H.L.-G.; Samaniego-Sánchez, C. Changes in the antioxidant properties of extra virgin olive oil after cooking typical mediterranean vegetables. Antioxidants 2019, 8, 246. [CrossRef] [PubMed] 
5. Martínez-González, M.A.; Salas-Salvadó, J.; Estruch, R.; Corella, D.; Fitó, M.; Ros, E. Benefits of the mediterranean diet: Insights from the predimed study. Prog. Cardiovasc. Dis. 2015, 58, 50-60. [CrossRef]

6. Cusimano, A.; Balasus, D.; Azzolina, A.; Augello, G.; Emma, M.R.; Di Sano, C.; Gramignoli, R.; Strom, S.C.; Mccubrey, J.A.; Montalto, G.; et al. Oleocanthal exerts antitumor effects on human liver and colon cancer cells through ROS generation. Int. J. Oncol. 2017, 51, 533-544. [CrossRef]

7. Francisco, V.; Ruiz-Fernández, C.; Lahera, V.; Lago, F.; Pino, J.; Skaltsounis, L.; González-Gay, M.A.; Mobasheri, A.; Gómez, R.; Scotece, M.; et al. Natural molecules for healthy lifestyles: Oleocanthal from extra virgin olive oil. J. Agric. Food Chem. 2019, 67, 3845-3853. [CrossRef]

8. Kiritsakis, A.; Shahidi, F. Olives and Olive Oil as Functional Foods; Shahidi, F., Kiritsakis, A., Eds.; John Wiley \& Sons, Ltd.: Chichester, UK, 2017; ISBN 9781119135340.

9. Marković, A.K.; Torić, J.; Barbarić, M.; Brala, C.J. Hydroxytyrosol, tyrosol and derivatives and their potential effects on human health. Molecules 2019, 24, 2001. [CrossRef]

10. Lozano-Castellón, J.; López-Yerena, A.; de Alvarenga, J.F.R.; del Castillo-Alba, J.R.; Vallverdú-Queralt, A.; Escribano-Ferrer, E.; Lamuela-Raventós, R.M. Health-promoting properties of oleocanthal and oleacein: Two secoiridoids from extra-virgin olive oil. Crit. Rev. Food Sci. Nutr. 2019, 1-7. [CrossRef]

11. Robles-Almazan, M.; Pulido-Moran, M.; Moreno-Fernandez, J.; Ramirez-Tortosa, C.; Rodriguez-Garcia, C.; Quiles, J.L.; Ramirez-Tortosa, M. Hydroxytyrosol: Bioavailability, toxicity, and clinical applications. Food Res. Int. 2018, 105, 654-667. [CrossRef]

12. Silva, S.; Sepodes, B.; Rocha, J.; Direito, R.; Fernandes, A.; Brites, D.; Freitas, M.; Fernandes, E.; Bronze, M.R.; Figueira, M.E. Protective effects of hydroxytyrosol-supplemented refined olive oil in animal models of acute inflammation and rheumatoid arthritis. J. Nutr. Biochem. 2015, 26, 360-368. [CrossRef]

13. Parkinson, L.; Keast, R. Oleocanthal, a phenolic derived from virgin olive oil: A review of the beneficial effects on inflammatory disease. Int. J. Mol. Sci. 2014, 15, 12323-12334. [CrossRef] [PubMed]

14. Cicerale, S.; Lucas, L.J.; Keast, R.S. Oleocanthal: A naturally occurring anti-inflammatory agent in virgin olive oil. In Olive Oil-Constituents, Quality, Health Properties and Bioconversions; InTech: Rijeka, Croatia, 2012. [CrossRef]

15. Cañuelo, A.; Gilbert-López, B.; Pacheco-Liñán, P.; Martínez-Lara, E.; Siles, E.; Miranda-Vizuete, A. Tyrosol, a main phenol present in extra virgin olive oil, increases lifespan and stress resistance in Caenorhabditis elegans. Mech. Ageing Dev. 2012, 133, 563-574. [CrossRef] [PubMed]

16. Rodríguez-Morató, J.; Boronat, A.; Kotronoulas, A.; Pujadas, M.; Pastor, A.; Olesti, E.; Pérez-Mañá, C.; Khymenets, O.; Fitó, M.; Farré, M.; et al. Metabolic disposition and biological significance of simple phenols of dietary origin: Hydroxytyrosol and tyrosol. Drug Metab. Rev. 2016, 48, 218-236. [CrossRef] [PubMed]

17. Bu, Y.; Rho, S.; Kim, J.; Kim, M.Y.; Lee, D.H.; Kim, S.Y.; Choi, H.; Kim, H. Neuroprotective effect of tyrosol on transient focal cerebral ischemia in rats. Neurosci. Lett. 2007, 414, 218-221. [CrossRef] [PubMed]

18. Di Benedetto, R.; Varì, R.; Scazzocchio, B.; Filesi, C.; Santangelo, C.; Giovannini, C.; Matarrese, P.; D'Archivio, M.; Masella, R. Tyrosol, the major extra virgin olive oil compound, restored intracellular antioxidant defences in spite of its weak antioxidative effectiveness. Nutr. Metab. Cardiovasc. Dis. 2007, 17, 535-545. [CrossRef]

19. Giovannini, C.; Straface, E.; Modesti, D.; Coni, E.; Cantafora, A.; De Vincenzi, M.; Malorni, W.; Masella, R. Tyrosol, the major olive oil biophenol, protects against oxidized-LDL-induced injury in Caco-2 cells. J. Nutr. 1999, 129, 1269-1277. [CrossRef]

20. Covas, M.I.; Miró-Casas, E.; Fitó, M.; Farré-Albadalejo, M.; Gimeno, E.; Marrugat, J.; De La Torre, R. Bioavailability of tyrosol, an antioxidant phenolic compound present in wine and olive oil, in humans. Drugs Exp. Clin. Res. 2003, 29, 203.

21. Lucas, L.; Russell, A.; Keast, R. Molecular mechanisms of inflammation. Anti-inflammatory benefits of virgin olive oil and the phenolic compound oleocanthal. Curr. Pharm. Des. 2011, 17, 754-768. [CrossRef]

22. Pang, K.L.; Chin, K.Y. The biological activities of oleocanthal from a molecular perspective. Nutrients 2018, 10, 570. [CrossRef]

23. Li, W.; Sperry, J.B.; Crowe, A.; Trojanowski, J.Q.; Smith, A.B.; Lee, V.M.Y. Inhibition of tau fibrillization by oleocanthal via reaction with the amino groups of tau. J. Neurochem. 2009, 110, 1339-1351. [CrossRef]

24. Des Gachons, C.P.; Uchida, K.; Bryant, B.; Shima, A.; Sperry, J.B.; Dankulich-Nagrudny, L.; Tominaga, M.; Smith, A.B.; Beauchamp, G.K.; Breslin, P.A.S. Unusual pungency from extra-virgin olive oil is attributable to restricted spatial expression of the receptor of oleocanthal. J. Neurosci. 2011, 31, 999-1009. [CrossRef] [PubMed] 
25. Iacono, A.; Gómez, R.; Sperry, J.; Conde, J.; Bianco, G.; Meli, R.; Gómez-Reino, J.J.; Smith, A.B.; Gualillo, O. Effect of oleocanthal and its derivatives on inflammatory response induced by lipopolysaccharide in a murine chondrocyte cell line. Arthritis Rheum. 2010, 62, 1675-1682. [CrossRef] [PubMed]

26. Agrawal, K.; Melliou, E.; Li, X.; Pedersen, T.L.; Wang, S.C.; Magiatis, P.; Newman, J.W.; Holt, R.R. Oleocanthal-rich extra virgin olive oil demonstrates acute anti-platelet effects in healthy men in a randomized trial. J. Funct. Foods 2017, 36, 84-93. [CrossRef] [PubMed]

27. Kotsiou, A.; Tesseromatis, C. Oleocanthal an extra-virgin olive oil bioactive component. J. Med. Plants Stud. 2017, 5, 95-100.

28. Sivakumar, G.; Briccoli-Bati, C.; Uccella, N. Demethyloleuropein and $\beta$-glucosidase activity in olive fruits. Biotechnol. J. 2007, 2, 381-385. [CrossRef] [PubMed]

29. De Medina, V.S.; Miho, H.; Melliou, E.; Magiatis, P.; Priego-Capote, F.; de Castro, M.D.L. Quantitative method for determination of oleocanthal and oleacein in virgin olive oils by liquid chromatography-tandem mass spectrometry. Talanta 2017, 162, 24-31. [CrossRef]

30. Obied, H.K.; Prenzler, P.D.; Ryan, D.; Servili, M.; Taticchi, A.; Esposto, S.; Robards, K. Biosynthesis and biotransformations of phenol-conjugated oleosidic secoiridoids from Olea europaea L. Nat. Prod. Rep. 2008, 25, 1167. [CrossRef]

31. Vougogiannopoulou, K.; Lemus, C.; Halabalaki, M.; Pergola, C.; Werz, O.; Smith, A.B.; Michel, S.; Skaltsounis, L.; Deguin, B. One-step semisynthesis of oleacein and the determination as a 5-lipoxygenase inhibitor. J. Nat. Prod. 2014, 77, 441-445. [CrossRef]

32. Gutierrez-Rosales, F.; Romero, M.P.; Casanovas, M.; Motilva, M.J.; Mínguez-Mosquera, M.I. Metabolites involved in oleuropein accumulation and degradation in fruits of olea europaea 1.: Hojiblanca and arbequina varieties. J. Agric. Food Chem. 2010, 58, 12924-12933. [CrossRef]

33. Fogli, S.; Arena, C.; Carpi, S.; Polini, B.; Bertini, S.; Digiacomo, M.; Gado, F.; Saba, A.; Saccomanni, G.; Breschi, M.C.; et al. Cytotoxic activity of oleocanthal isolated from virgin olive oil on human melanoma cells. Nutr. Cancer 2016, 68, 873-877. [CrossRef]

34. Siddique, A.B.; Ebrahim, H.; Mohyeldin, M.; Qusa, M.; Batarseh, Y.; Fayyad, A.; Tajmim, A.; Nazzal, S.; Kaddoumi, A.; El Sayed, K. Novel liquid-liquid extraction and self-emulsion methods for simplified isolation of extra-virgin olive oil phenolics with emphasis on (-)-oleocanthal and its oral anti-breast cancer activity. PLoS ONE 2019, 14, e0214798. [CrossRef] [PubMed]

35. Angelis, A.; Hamzaoui, M.; Aligiannis, N.; Nikou, T.; Michailidis, D.; Gerolimatos, P.; Termentzi, A.; Hubert, J.; Halabalaki, M.; Renault, J.-H.; et al. An integrated process for the recovery of high added-value compounds from olive oil using solid support free liquid-liquid extraction and chromatography techniques. J. Chromatogr. A 2017, 1491, 126-136. [CrossRef] [PubMed]

36. Adhami, H.-R.; Zehl, M.; Dangl, C.; Dorfmeister, D.; Stadler, M.; Urban, E.; Hewitson, P.; Ignatova, S.; Krenn, L. Preparative isolation of oleocanthal, tyrosol, and hydroxytyrosol from olive oil by HPCCC. Food Chem. 2015, 170, 154-159. [CrossRef] [PubMed]

37. Tasioula-Margari, M.; Tsabolatidou, E. Extraction, separation, and identification of phenolic compounds in virgin olive oil by HPLC-DAD and HPLC-MS. Antioxidants 2015, 4, 548-562. [CrossRef]

38. Bianco, A.; Buiarelli, F.; Cartoni, G.; Coccioli, F.; Muzzalupo, I.; Polidori, A.; Uccella, N. Analysis by Hplc-Ms/Ms of biophenolic components in olives and oils. Anal. Lett. 2001, 34, 1033-1051. [CrossRef]

39. Luque-Muñoz, A.; Tapia, R.; Haidour, A.; Justicia, J.; Cuerva,J.M. Direct determination of phenolic secoiridoids in olive oil by ultra-high performance liquid chromatography-triple quadruple mass spectrometry analysis. Sci. Rep. 2019, 9, 15545. [CrossRef]

40. Bonacci, S.; Di Gioia, M.L.; Costanzo, P.; Maiuolo, L.; Tallarico, S.; Nardi, M. Natural deep eutectic solvent as extraction media for the main phenolic compounds from olive oil processing wastes. Antioxidants 2020, 9, 513. [CrossRef]

41. Fanali, C.; Posta, S.D.; Dugo, L.; Russo, M.; Gentili, A.; Mondello, L.; De Gara, L. Application of deep eutectic solvents for the extraction of phenolic compounds from extra-virgin olive oil. Electrophoresis 2020. [CrossRef]

42. Shabani, E.; Zappi, D.; Berisha, L.; Dini, D.; Antonelli, M.L.; Sadun, C. Deep eutectic solvents (DES) as green extraction media for antioxidants electrochemical quantification in extra-virgin olive oils. Talanta 2020, 215, 120880. [CrossRef] 
43. Paradiso, V.M.; Clemente, A.; Summo, C.; Pasqualone, A.; Caponio, F. Extraction of phenolic compounds from extra virgin olive oil by a natural deep eutectic solvent: Data on UV absorption of the extracts. Data Br. 2016, 8, 553-556. [CrossRef]

44. García, A.; Rodríguez-Juan, E.; Rodríguez-Gutiérrez, G.; Rios, J.J.; Fernández-Bolaños, J. Extraction of phenolic compounds from virgin olive oil by deep eutectic solvents (DESs). Food Chem. 2016, 197, 554-561. [CrossRef] [PubMed]

45. Polini, B.; Digiacomo, M.; Carpi, S.; Bertini, S.; Gado, F.; Saccomanni, G.; Macchia, M.; Nieri, P.; Manera, C.; Fogli, S. Oleocanthal and oleacein contribute to the in vitro therapeutic potential of extra virgin oil-derived extracts in non-melanoma skin cancer. Toxicol. In Vitro 2018, 52, 243-250. [CrossRef] [PubMed]

Sample Availability: Samples of the studied compounds are available from the authors.

(C) 2020 by the authors. Licensee MDPI, Basel, Switzerland. This article is an open access article distributed under the terms and conditions of the Creative Commons Attribution (CC BY) license (http://creativecommons.org/licenses/by/4.0/). 